\title{
Prevention of sensory disorders in diabetic Sprague-Dawley rats by aldose reductase inhibition or treatment with ciliary neurotrophic factor
}

\author{
N. A. Calcutt ${ }^{1}$ J. D. Freshwater ${ }^{1}$ - A. P. Mizisin ${ }^{1}$ \\ ${ }^{1}$ Department of Pathology, University of California, San Diego, La Jolla, USA
}

\begin{abstract}
Aims/hypothesis. Sensory neuropathy in diabetic patients frequently presents itself as progressive loss of thermal perception, while some patients describe concurrent spontaneous pain, allodynia or hyperalgesia. Diabetic rats develop thermal hypoalgesia and tactile allodynia by unknown mechanisms. We investigated whether sensory disorders in rats were related to glucose metabolism by aldose reductase. We also explored the therapeutic potential of exogenous neurotrophic factors.

Methods. Behavioural assessments of thermal and tactile sensitivity were performed in normal rats and in rats with streptozotocin-induced diabetes. Some of the rats were treated with insulin, aldose reductase inhibitors, ciliary neurotrophic factor or brain-derived neurotrophic factor.

Results. Thermal hypoalgesia was present after 8 weeks of diabetes and was prevented by insulin treatment, which maintained normoglycaemia, by the aldose reductase inhibitor Statil or by ciliary neurotrophic factor. Brain-derived neurotrophic factor did not have
\end{abstract}

an effect. When diabetic rats were tested after shorter durations of diabetes, they showed transient thermal hyperalgesia after 4 weeks which progressed to thermal hypoalgesia after 8 weeks. The aldose reductase inhibitor IDD 676 (Lidorestat), given from the onset of diabetes, prevented the development of thermal hyperalgesia and also stopped progression to thermal hypoalgesia when delivered in the last 4 weeks of an 8 -week period of diabetes. Tactile allodynia was not prevented by neurotrophic factor or aldose reductase inhibitor treatment.

Conclusions/interpretation. Transient thermal hyperalgesia and subsequent progressive thermal hypoalgesia occur in diabetic rats secondary to exaggerated flux through the polyol pathway. A depletion of ciliary neurotrophic factor mediated by the polyol pathway may be involved in the aetiology of thermal hypoalgesia.

Keywords Aldose reductase - Allodynia .

Brain-derived neurotrophic factor .

Ciliary neurotrophic factor - Diabetic neuropathy ·

Hyperalgesia $\cdot$ Hypoalgesia $\cdot$ Pain · Peripheral nerve
Received: 17 October 2003 / Accepted: 21 November 2003 Published online: 13 March 2004

C) Springer-Verlag 2004

N. A. Calcutt (-)

Department of Pathology, University of California,

San Diego, La Jolla, CA 92093-0612, USA

E-mail: ncalcutt@ucsd.edu

Abbreviations: CNTF, ciliary neurotrophic factor $\cdot$ BDNF, brain-derived neurotrophic factor

\section{Introduction}

Progressive loss of thermal perception and thermal pain perception thresholds in the extremities are amongst the most frequently encountered manifestations of diabetic neuropathy. Loss of sensation in the feet is particularly problematic, and when combined with microvascular disease and impaired wound healing, is responsible for initiating a pathological development that makes diabetes the leading cause of lower limb amputation in western societies [1]. A proportion of patients with sensory loss also describe a concomi- 
tant state of pain which can be spontaneous or touchevoked. While it is clear that maintaining good glycaemic control is a primary goal in efforts to restrict the onset and progression of diabetic neuropathy, understanding the aetiological mechanisms leading to sensory loss and neuropathic pain may provide alternative targets for therapeutic intervention which could complement efforts to maintain normoglycaemia.

Diabetic rodents develop a number of neurochemical, electrophysiological and behavioural disorders that have prompted the use of these animals in modelling the effects of short-term diabetes on the peripheral nervous system. These disorders include behavioural indices of allodynia in response to light touch and of hyperalgesia in response to noxious chemical stimuli [2]. Behavioural responses to thermal stimuli have also been studied in diabetic rodents, but no clear picture has emerged. Using the tail-flick test of spinally mediated reflexes, diabetic mice have been described with increased [3] or unchanged [4] response times, while diabetic rats are reported to have increased [5] or decreased [6, 7] response times. Heating the plantar surface of the paw to evoke withdrawal responses has also provided conflicting results, with both hypoalgesia and hyperalgesia reported $[8,9,10$, $11,12,13]$. That no simple pattern of response has yet emerged may reflect subtle differences in the nature of the tests and the animal models studied.

In our previous studies using adult female streptozotocin-diabetic rats, we have routinely found that thermal hypoalgesia develops in the hind paw after approximately 8 weeks $[14,15,16,17]$ when using a system that selectively activates $\mathrm{C}$ fibres by increasing contact surface temperature from 30 to $50^{\circ} \mathrm{C}$ over a 20 -second period [18]. Thermal hypoalgesia was prevented by treating rats with a neuroactive peptide that did not alter the general physiological condition of the animals $[15,16]$, indicating that thermal hypoalgesia is unlikely to be a consequence of cachexia or altered thermal transduction properties of the skin. The mechanism by which this peptide exerts its physiological effects is not yet known, so these findings do not help explain the cause of the underlying thermal hypoalgesia. In the present series of experiments, we sought to define the primary cause of thermal hypoalgesia and tactile allodynia by treating diabetic rats with insulin to maintain normoglycaemia, or with aldose reductase inhibitors to prevent glucose metabolism through the first step of the polyol pathway. We also investigated the therapeutic potential of members of two distinct classes of neurotrophic factors, because loss of neurotrophic support has been implicated in the development of diabetic neuropathy and may be a downstream consequence of exaggerated sugar metabolism by aldose reductase [19].

\section{Materials and methods}

Animals, drugs and treatments. All studies were performed under a protocol approved by the local Animal Subjects Committee and in accordance with the National Institutes for Health 'Principles of Laboratory Animal Care, 1985 revised version'. Type 1 diabetes was induced by delivering a single intraperitoneal injection of streptozotocin (Sigma, St. Louis, Mich., USA) dissolved in $0.9 \%$ sterile saline at a dose of $50 \mathrm{mg} / \mathrm{kg}$ body weight to fasted adult female Sprague-Dawley rats (Harlan Industries, San Diego, Calif., USA). After 4 days, glucose levels were measured in aliquots of blood obtained by tail prick using a strip-operated reflectance meter. Only streptozotocin-injected animals with a blood sugar level of above $15 \mathrm{mmol} / \mathrm{l}$ were included in the study as being diabetic. All diabetic rats were monitored daily and animals showing behavioural discomfort, such as depression of motor activity or cachexia, were removed from the study. At the end of each study, blood was obtained by cardiac puncture and plasma glucose levels were measured using a spectrophotometric assay kit (Trinder Assay Kit, Sigma).

Insulin treatment of diabetic rats was carried out using slow-dissolving pellets that deliver approximately 2 to $4 \mathrm{U}$ insulin per day (Linshin, Scarborough, Ont, Canada). Pellets were implanted under the skin at the onset of hyperglycaemia and blood glucose was checked weekly in samples obtained by tail prick. When blood sugar levels increased to above $15 \mathrm{mmol} / \mathrm{l}$, implants were replaced. The aldose reductase inhibitors Statil (Astra-Zeneca, Macclesfield, UK) and IDD 676 (Institute for Diabetes Discovery, Branford, Conn., USA) were delivered by daily oral gavage at doses of 50 and $10 \mathrm{mg} / \mathrm{kg}$ body weight respectively. These doses were selected because of their ability to inhibit polyol accumulation, restore nerve levels of neurotrophic factors and protect the nerve function of diabetic rats in previously published observations $[19,20]$ and preliminary studies (N.A. Calcutt, unpublished observations). Efficacy of treatment was confirmed by measuring sciatic nerve sorbitol content at the end of the study by gas chromatography [2]. Control animals received an equivalent volume of vehicle (phosphate-buffered saline with 1 drop of Tween 80 per $\mathrm{ml}$ buffer). Human recombinant ciliary neurotrophic factor (CNTF, $1 \mathrm{mg} / \mathrm{kg}$ ) and brain-derived neurotrophic factor (BDNF, $15 \mathrm{mg} / \mathrm{kg}$ ) were delivered three times weekly by subcutaneous injection beginning at the onset of hyperglycaemia. The two neurotrophic factors were provided by Regeneron (Tarrytown, N.Y., USA) and the doses were selected on the evidence of previously published findings [21] and preliminary studies (A.P. Mizisin, unpublished observations) showing the ability of these factors to protect nerve function in hyperglycaemic rats. Behavioural studies were performed 24 to 30 hours after the last drug treatment to prevent distortion of results through transient effects of the treatment [16].

Paw thermal response latency. Because preliminary studies suggested that frequent repeated testing of rats over a number of weeks leads to habituation (N.A. Calcutt, unpublished observations), all rats underwent testing at only one time point within a study. For testing, rats were placed in a plexiglass enclosure on the glass floor of a thermal testing apparatus (UARD, San Diego, Calif., USA). The glass floor was maintained at $30^{\circ} \mathrm{C}$ and rats were allowed to acclimatise for $15 \mathrm{~min}$. The right hind paw was then exposed to a heat source directed from below the glass floor and focused on the centre of the paw. The latency from the initiation of heating to when the paw was deliberately withdrawn from the glass surface was recorded using the automated timer of the thermal testing ap- 
Table 1. Body weight, plasma glucose and tactile responses in all rats

\begin{tabular}{|c|c|c|c|c|}
\hline Group & $n$ & Body weight (g) & Plasma glucose $(\mathrm{mmol} / \mathrm{l})$ & $50 \%$ response threshold $(\mathrm{g})$ \\
\hline Control & 6 & $300 \pm 6$ & $5.2 \pm 1.9$ & $13.5(8.0-15.0)$ \\
\hline Control + CNTF & 6 & $295 \pm 8$ & $4.2 \pm 1.4$ & $8.3(1.9-15.0)$ \\
\hline Diabetic (8 weeks) & 6 & $212 \pm 11 *$ & $46.5 \pm 1.6^{*}$ & $2.2(1.6-2.8)^{*}$ \\
\hline Diabetic + CNTF & 6 & $208 \pm 7 *$ & $43.6 \pm 0.9 *$ & $3.2(1.6-4.3) *$ \\
\hline Diabetic + BDNF & 5 & $206 \pm 9 *$ & $46.4 \pm 3.1 *$ & $2.8(2.2-3.3) *$ \\
\hline Diabetic (8 weeks) & 10 & $189 \pm 6^{*}$ & $24.6 \pm 1.0 *$ & NM \\
\hline Diabetic + Statil & 6 & $186 \pm 9 *$ & $26.1 \pm 1.0 *$ & NM \\
\hline Control & 6 & $250 \pm 4$ & $6.2 \pm 1.0$ & $7.5(5.6-15.0)$ \\
\hline Diabetic (4 weeks) & 8 & $205 \pm 4 *$ & $36.3 \pm 1.9 *$ & $2.6(1.6-4.5)^{*}$ \\
\hline Diabetic + IDD 676 & 6 & $201 \pm 7 *$ & $32.2 \pm 2.1 *$ & $2.3(1.6-4.0) *$ \\
\hline Control & 6 & $267 \pm 4$ & $8.4 \pm 1.0$ & $10.0(6.6-15.0)$ \\
\hline
\end{tabular}

Data are means \pm SEM or median (range) as appropriate. Statistical comparisons between controls and all other groups were carried out using one-way ANOVA followed by Dunnett's test for parametric data, or the Kruskal-Wallis test followed by Dunn's test for non-parametric data.

paratus. The right hind paw of each rat was tested three times at 5-minute intervals following an initial acclimatisation test, and the median of the three tests was used for statistical analysis. On each day that tests were done, the change in temperature of the glass floor over a 20 -second period was also recorded to confirm that it increased at the rate of $1^{\circ} \mathrm{C}$ per second.

Paw tactile response threshold. Because repeated testing of diabetic rats can distort behavioural responses to therapeutics [16], animals were only tested at the end of the treatment protocol. Rats were transferred to a testing cage with a wire mesh bottom and allowed to acclimatise for 10 to $15 \mathrm{~min}$. Von Frey filaments (Stoelting, Wood Dale, Ill., USA) were used to determine the $50 \%$ mechanical threshold for foot withdrawal. A series of filaments, starting with one that had a buckling weight of $2.0 \mathrm{~g}$, were applied in sequence to the plantar surface of the right hind paw with a pressure that caused the filament to buckle. Lifting of the paw was recorded as a positive response and a lighter filament was chosen for the next measurement. If there was no response after 5 seconds, the next heaviest filament was used afterwards. This method was continued until four measurements had been made after an initial change in the behaviour or until five consecutive negative (15 g) or four consecutive positive $(0.25 \mathrm{~g})$ responses had occurred. The resulting sequence of positive and negative scores was used to interpolate the $50 \%$ response threshold exactly as described elsewhere [22].

Statistical analysis. Parametric data are presented as the group means \pm SEM, with statistical comparisons between groups made using one-way ANOVA and subsequent identification of groups significantly different from controls carried out using Dunnett's test. Non-parametric data are presented as the group median and range, with statistical comparisons between groups made using the Kruskal-Wallis test, and groups significantly different from controls being subsequently identified by Dunn's test. A $p$ value of $<0.05$ was considered statistically significant. The number of animals per group $(n)$ is given in Table 1 .
BDNF, brain-derived neurotrophic factor; CNTF, ciliary neurotrophic factor; NM, not measured. ${ }^{*} p<0.05$ vs control

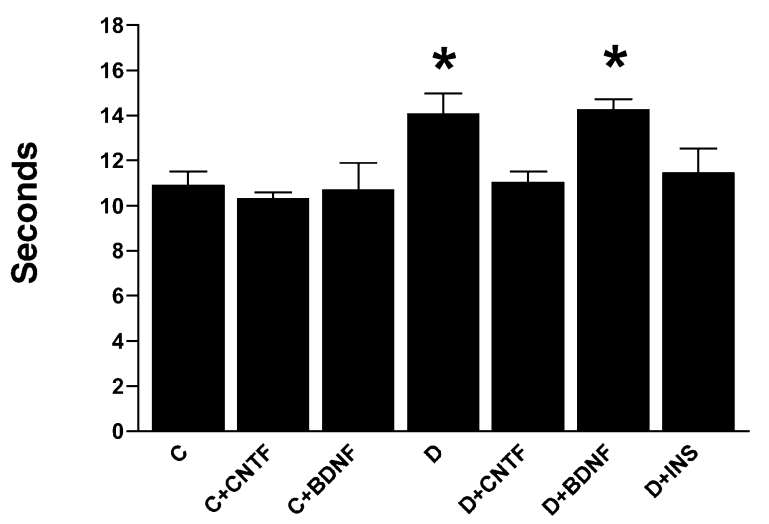

Fig. 1. Hind paw thermal response latency after 8 weeks of treatment with vehicle, ciliary neurotrophic factor (CNTF), brain-derived neurotrophic factor (BDNF) or insulin (INS) in control (C) and diabetic (D) rats. Data are means \pm SEM. Statistical analysis was carried out using one-way ANOVA with Dunnett's test. $* p<0.05$ vs control

\section{Results}

\section{Paw thermal sensitivity}

Insulin or neurotrophic factor treatment. Leaving streptozotocin-induced diabetes untreated for 8 weeks resulted in weight loss, hyperglycaemia and thermal hypoalgesia [all $p<0.05$ compared with untreated controls (Table 1, Fig. 1)]. Insulin treatment of streptozotocin-injected rats, beginning after confirmation of hyperglycaemia and lasting for 8 weeks, normalised blood sugar levels and body weight at the end of this period when compared to control rats (Table 1). Insulin treatment of diabetic rats also maintained ther- 
mal response latencies at values that were not different from those of untreated controls (Fig. 1).

Treating control rats with CNTF or BDNF for the duration of the study did not affect body weight, plasma glucose levels or thermal response latency (Table 1 and Fig. 1). Hyperglycaemia and body weight of diabetic rats were also unaffected by BDNF or CNTF treatment (Table 1). Diabetic rats treated with CNTF had thermal response latencies that were not significantly different from untreated controls. In contrast, rats treated with BDNF had similar thermal response latencies to untreated diabetic rats and were significantly hypoalgesic $(p<0.05)$ compared to untreated controls (Fig. 1).

Aldose reductase inhibition. Efficacy of aldose reductase inhibition was indicated by the reduction of sorbitol accumulation in the sciatic nerve of Statil-treated diabetic rats $(0.2 \pm 0.2 \mathrm{nmol} / \mathrm{mg}$ dry weight $)$ when compared to vehicle-treated diabetic rats $(1.5 \pm 0.4 \mathrm{nmol} / \mathrm{mg}$ dry weight). Treatment with Statil for 8 weeks of diabetes did not affect the body weight or hyperglycaemia of diabetic rats (Table 1). Thermal response latencies were significantly increased $(p<0.01)$ in vehicle-treated diabetic rats when compared with controls and were significantly lower than the untreated diabetic rats (Fig. 2), whereas those of Statil-treated diabetic rats were not different from controls.

To determine whether aldose reductase inhibition was also effective in shorter prevention and reversal treatments, we performed two further studies using the aldose reductase inhibitor IDD 676. In the first study, thermal response latency was measured after a 4-week period in control rats, untreated diabetic rats and diabetic rats treated daily with IDD 676 for the duration of diabetes. At this time point, diabetic rats had significant ( $p<0.05$ vs control) hyperalgesia, but it was completely prevented by IDD 676 (Fig. 3). In the second study, diabetic rats were either left untreated for 8 weeks or received IDD 676 for the last 4 weeks only. After 8 weeks of untreated diabetes, rats had significant thermal hypoalgesia ( $p<0.05$ vs control). In contrast, the diabetic rats treated with IDD 676 had thermal response latencies that were not different from controls (Fig. 3). Treatment with IDD 676 did not affect hyperglycaemia or body weight in diabetic rats in either of these studies (Table 1), and no sorbitol was detected in the sciatic nerve of these animals (limit of detection was $1.8 \mathrm{nmol}$ per tissue sample), indicating that aldose reductase inhibition was effective.

\section{Paw tactile responses}

Diabetic rats showed tactile allodynia $(p<0.05$ vs controls), which was prevented by insulin but not by treatment with CNTF, BDNF or the aldose reductase inhibitor IDD 676 (Table 1).

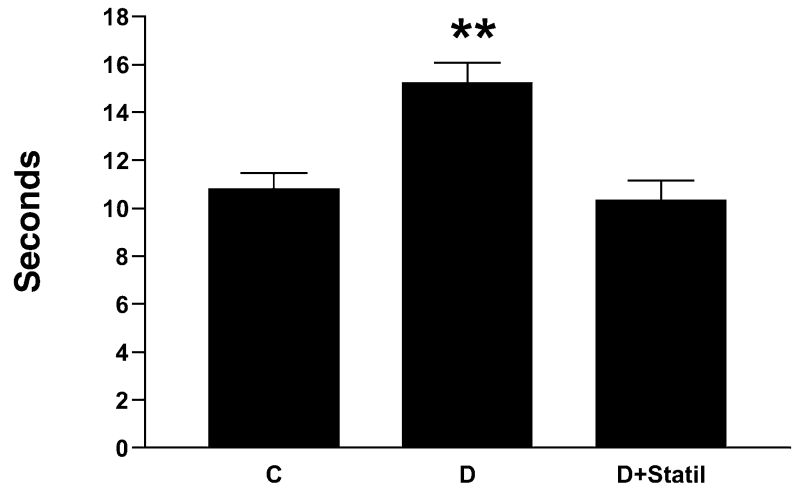

Fig. 2. Hind paw thermal response latency after 8 weeks in control (C), diabetic (D) and Statil-treated diabetic (D+Statil) rats (Statil treatment, $\left.50 \mathrm{mg} \cdot \mathrm{kg}^{-1} \cdot \mathrm{day}^{-1}\right)$. Data are means \pm SEM. Statistical analysis was carried out using one-way ANOVA with Dunnett's test. $* * p<0.01$ vs control

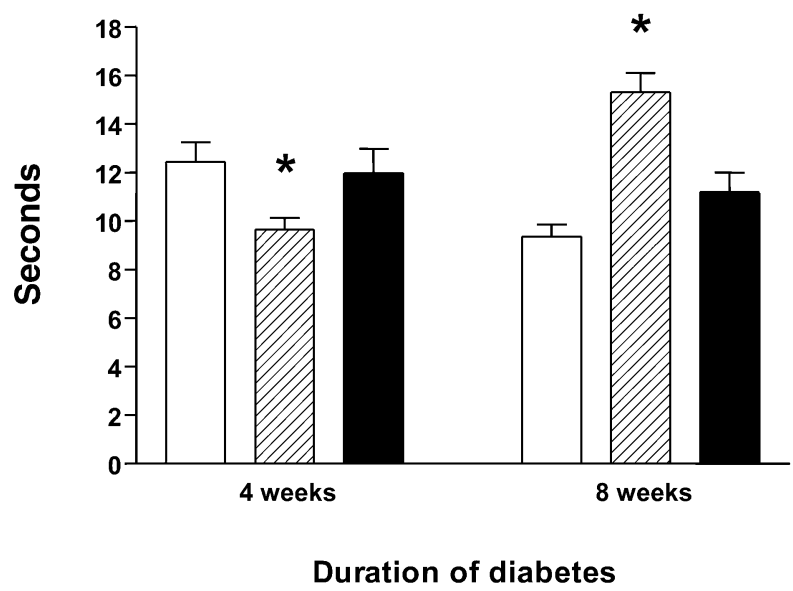

Fig. 3. Hind paw thermal response latency after 4 and 8 weeks in control (white bars), diabetic (hatched bars) and IDD 676-treated diabetic (black bars) rats (IDD 676 treatment, $\left.10 \mathrm{mg} \cdot \mathrm{kg}^{-1} \cdot \mathrm{day}^{-1}\right)$. Treatment was from week 0 to week 4 , or from week 4 to week 8 . Data are means \pm SEM. Statistical analysis was carried out using one-way ANOVA with Dunnett's test. $* p<0.05$ vs control

\section{Discussion}

Abnormal perception of heat and heat-induced pain occurs in diabetic patients and can range from thermal hyperalgesia in early stages of neuropathy to the progressive thermal hypoalgesia associated with degenerative neuropathy, which includes the loss of the epidermal $\mathrm{C}$ fibre terminals involved in thermal nociception $[23,24,25]$. The thermal response latency represents a similar test in diabetic rodents, but it is not yet clear whether loss of epidermal $\mathrm{C}$ fibres occurs in diabetic rats with thermal hypoalgesia. Indeed, the only morphological study reported to date showed no loss of epidermal immunostaining for the pan-axonal marker, Protein Gene Product 9.5, or the neuropeptide, calcitonin gene-related peptide, after 8 weeks of diabetes [26], although the thermal responsiveness of 
these rats was not recorded. Diabetic mice did show loss of epidermal fibres [27], but no correlation to thermal nociception has yet been observed. Another possibility is that thermal hypoalgesia is related to the diabetes-induced disruption of sensory nerve phenotype. This is illustrated by the impaired synthesis, axonal transport and evoked release of neuropeptides $[17,28,29,30,31]$, and occurs as a response to diminished neurotrophic support [32, 33]. Interestingly, agents preventing thermal hypoalgesia in diabetic rats have been reported to also protect nerve neuropeptide levels in diabetic rats $[16,28,29]$ and to enhance neuropeptide levels in sensory ganglia of adult rats [34].

In the present and previous studies, we have found there to be thermal hypoalgesia in rats after 8 weeks of diabetes [14, 15, 16, 17]. Hypoalgesia is not likely to be caused by direct streptozotocin-induced neurotoxicity, as it was prevented by the insulin therapy that maintained normoglycaemia. That two different aldose reductase inhibitors were effective suggests that glucose metabolism by aldose reductase is involved in the aetiology of thermal hypoalgesia, as it is found in a host of peripheral nerve disorders induced by diabetes [35]. The progression from increased glucose metabolism in cells that express aldose reductase to the dysfunction of thermal nociception mediated by $\mathrm{C}$ fibres remains to be clarified. One plausible intermediate in this progression could be CNTF deficiency. The bioactivity of this Schwann-cell-derived neurotrophic factor, as well as the amount of protein are reduced in the peripheral nerve of diabetic rats, and aldose reductase inhibition can increase CNTF in the nerve of hyperglycaemic rats $[19,36]$. In the present study, CNTF treatment prevented thermal hypoalgesia in diabetic rats without affecting responses in control rats. This distinguishes CNTF from members of the neurotrophin family of neurotrophic factors, which either have no effect on thermal discrimination in diabetes (as we found using BDNF with a dose that protects large fibres from hyperglycaemia [21]), or have hyperalgesic effects on control and diabetic animals, as reported for nerve growth factor [5]. CNTF has been largely associated with peripheral motor and sensory neuron survival and regeneration after injury [37, 38 ], rather than with the provision of ongoing neurotrophic support in the adult nervous system. Nevertheless, the CNTFR $\alpha$ receptor for CNTF is expressed by both large and small cell bodies in adult sensory ganglia and is detected in peripheral nerve axons [39]. The direct effects of exogenously administered CNTF on peripheral $\mathrm{C}$ fibres may play a role in the protection of thermal nociceptive function in diabetic rats, although indirect effects mediated via actions on other cell types cannot yet be discounted.

From our studies, designed to examine the effects of aldose reductase inhibition, we found that 4 weeks of untreated diabetes induced thermal hyperalgesia, which ultimately progressed to hypoalgesia. One pre- vious study has reported a similar progression [40] and this time dependence may contribute to the variable findings in the literature. Thermal hyperalgesia was prevented by aldose reductase inhibition, which is consistent with the efficacy of these agents observed in other rat models of diabetic hyperalgesia, such as that which occurs during the formalin test [41, 42]. Interestingly, aldose reductase inhibition also normalised thermal responses when given between weeks 4 and 8 of diabetes, suggesting a reversal of established hyperalgesia and prevention of the progression to thermal hypoalgesia. Electrophysiological correlates to the hyperalgesia seen in short-term diabetes may include altered sensitivity and firing patterns in primary afferents [43, 44, 45] or spontaneous activity and enhanced nociceptive processing in the spinal cord $[17,46,47,48]$. It remains to be seen whether these potential physiological and neurochemical indices of behavioural hyperalgesia are also prevented by aldose reductase inhibition.

The tactile allodynia present in diabetic rats appears to have an aetiology that is distinct from that underlying formalin hyperalgesia and abnormal thermal nociception. Allodynia in diabetic rats is not dependent on the function of small unmyelinated $C$ fibres [44] and is usually associated with large myelinated sensory fibres. Numerous agents that interfere with sensory processing have been shown to temporarily ameliorate tactile allodynia in diabetic rats [49], while prolonged tight glycaemic control with insulin prevented the disorder from developing [2]. The lack of efficacy of aldose reductase inhibitors is particularly surprising given their widely reported actions on other structural and functional disorders of large myelinated fibres in diabetic rats [35]. The recent report that direct local exposure of the dorsal root ganglion of normal rats to higher glucose levels induces tactile allodynia [50] suggests that hyperglycaemia, rather than insulin deficiency, may be involved. In light of this, consequences of hyperglycaemia that are not solely dependent on glucose metabolism by aldose reductase, such as non-enzymatic glycosylation or oxidative stress [46], may need further exploration.

In summary, we have described a progression from transient thermal hyperalgesia to thermal hypoalgesia in Type 1 diabetic rats which is related to increased glucose metabolism by aldose reductase. The efficacy of aldose reductase inhibitors and CNTF replacement in preventing thermal hypoalgesia provides insight into the aetiological mechanisms underlying this disorder and may suggest therapeutic approaches to treating the progressive sensory loss that occurs in diabetic neuropathy.

Acknowledgements. Our thanks to Dr Janet Sredy (Institute for Diabetes Discovery), Dr Don Mirlees (Astra-Zeneca) and Dr Ronald Lindsay (Regeneron) for providing the IDD 676, the Statil and the neurotrophic factors CNTF and BDNF respectively. This study was supported by the Institute for Diabetes Discovery and National Institutes for Health grants NS38855 and DK57629. 


\section{References}

1. Shaw JE, Boulton AJ (1997) The pathogenesis of diabetic foot problems: an overview. Diabetes 46 [Suppl 2]:S58S61

2. Calcutt NA, Jorge MC, Yaksh TL, Chaplan SR (1996) Tactile allodynia and formalin hyperalgesia in streptozotocin-diabetic rats: effects of insulin, aldose reductase inhibition and lidocaine. Pain 68:293-299

3. Levine AS, Morley JE, Wilcox G, Brown DM, Handwerger BS (1982) Tail pinch behavior and analgesia in diabetic mice. Physiol Behav 28:39-43

4. Kamei J, Ohhashi Y, Aoki T, Kasuya Y (1991) Streptozotocin-induced diabetes in mice reduces the nociceptive threshold, as recognized after application of noxious mechanical stimuli but not of thermal stimuli. Pharmacol Biochem Behav 39:541-544

5. Apfel SC, Arezzo JC, Brownlee M, Federoff H, Kessler JA (1994) NGF administration protects against experimental diabetic sensory neuropathy. Brain Res 634:7-12

6. Lee JH, McCarty R (1992) Pain threshold in diabetic rats: effects of good versus poor diabetic control. Pain 50:231236

7. Courteix C, Eschalier A, Lavarenne J (1993) Streptozocininduced diabetic rats: behavioural evidence for a model of chronic pain. Pain 53:81-88

8. Chu PC, Lin MT, Shian LR, Leu SY (1986) Alterations in physiologic functions and in brain monoamine content in streptozocin-diabetic rats. Diabetes 35:481-485

9. Forman LJ, Estilow S, Lewis M, Vasilenko P (1986) Streptozocin diabetes alters immunoreactive beta-endorphin levels and pain perception after $8 \mathrm{wk}$ in female rats. Diabetes 35:1309-1313

10. Fox A, Eastwood C, Gentry C, Manning D, Urban L (1999) Critical evaluation of the streptozotocin model of painful diabetic neuropathy in the rat. Pain 81:307316

11. Piercy V, Banner SE, Bhattacharyya A et al. (1999) Thermal, but not mechanical, nociceptive behavior is altered in the Zucker Diabetic Fatty rat and is independent of glycemic status. J Diabetes Complications 13:163169

12. Cameron NE, Tuck Z, McCabe L, Cotter MA (2001) Effect of the hydroxyl radical scavenger, dimethylthiourea, on peripheral nerve tissue perfusion, conduction velocity and nociception in experimental diabetes. Diabetologia 44:1161-1169

13. Zhang W, Slusher B, Murakawa Y et al. (2002) GCPII (NAALADase) inhibition prevents long-term diabetic neuropathy in type 1 diabetic BB/Wor rats. J Neurol Sci 194:21-28

14. Calcutt NA, Dines KC, Cesena RM (1998) Effects of the peptide HP228 on nerve disorders in diabetic rats. Metabolism 47:650-656

15. Calcutt NA, Campana WM, Eskeland NL et al. (1999) Prosaposin gene expression and the efficacy of a prosaposin-derived peptide in preventing structural and functional disorders of peripheral nerve in diabetic rats. J Neuropathol Exp Neurol 58:628-636

16. Calcutt NA, Freshwater JD, O'Brien JS (2000) Protection of sensory function and antihyperalgesic properties of a prosaposin-derived peptide in diabetic rats. Anesthesiology 93:1271-1278

17. Calcutt NA, Stiller C, Gustafsson H, Malmberg AB (2000) Elevated substance-P-like immunoreactivity levels in spinal dialysates during the formalin test in normal and diabetic rats. Brain Res 856:20-27
18. Yeomans DC, Proudfit HK (1996) Nociceptive responses to high and low rates of noxious cutaneous heating are mediated by different nociceptors in the rat: electrophysiological evidence. Pain 68:141-150

19. Mizisin AP, Calcutt NA, DiStefano PS, Acheson A, Longo FM (1997) Aldose reductase inhibition increases CNTFlike bioactivity and protein in sciatic nerves from galactose-fed and normal rats. Diabetes 46:647-652

20. Calcutt NA, Mizisin AP, Kalichman MW (1994) Aldose reductase inhibition, Doppler flux and conduction in diabetic rats nerve. Eur J Pharmacol 251:27-33

21. Mizisin AP, Bache M, DiStefano PS, Acheson A, Lindsay RM, Calcutt NA (1997) BDNF attenuates functional and structural disorders in nerves of galactose-fed rats. J Neuropath Exp Neurol 56:1290-1301

22. Chaplan SR, Bach FW, Pogrel JW, Chung JM, Yaksh TL (1994) Quantitative assessment of tactile allodynia in the rat paw. J Neurosci Meth 53:55-63

23. Dyck PJ, Dyck PJ, Larson TS, O'Brien PC, Velosa JA (2000) Patterns of quantitative sensation testing of hypoesthesia and hyperalgesia are predictive of diabetic polyneuropathy: a study of three cohorts. Nerve growth factor study group. Diabetes Care 23:510-517

24. Levy DM, Karanth SS, Springall DR, Polak JM (1989) Depletion of cutaneous nerves and neuropeptides in diabetes mellitus: an immunocytochemical study. Diabetologia 32:427-433

25. Kennedy WR, Wendelschafer-Crabb G, Johnson T (1996) Quantitation of epidermal nerves in diabetic neuropathy. Neurology 47:1042-1048

26. Karanth SS, Springall DR, Francavilla S, Mirrlees DJ, Polak JM (1990) Early increase in CGRP- and VIP-immunoreactive nerves in the skin of streptozotocin-induced diabetic rats. Histochemistry 94:659-666

27. Christianson JA, Riekhof JT, Wright DE (2003) Restorative effects of neurotrophin treatment on diabetes-induced cutaneous axon loss in mice. Exp Neurol 179:188-199

28. Robinson JP, Willars GB, Tomlinson DR, Keen P (1987) Axonal transport and tissue contents of substance $\mathrm{P}$ in rats with long-term streptozotocin-diabetes. Effects of the aldose reductase inhibitor 'statil'. Brain Res 426:339-348

29. Diemel LT, Stevens EJ, Willars GB, Tomlinson DR (1992) Depletion of substance P and calcitonin gene-related peptide in sciatic nerve of rats with experimental diabetes; effects of insulin and aldose reductase inhibition. Neurosci Lett 137:253-256

30. Calcutt NA, Chen P, Hua XY (1998) Effects of diabetes on tissue content and evoked release of calcitonin gene-related peptide-like immunoreactivity from rat sensory nerves. Neurosci Lett 254:129-132

31. Garrett NE, Malcangio M, Dewhurst M, Tomlinson DR (1997) alpha-Lipoic acid corrects neuropeptide deficits in diabetic rats via induction of trophic support. Neurosci Lett 222:191-194

32. Diemel LT, Brewster WJ, Fernyhough P, Tomlinson DR (1994) Expression of neuropeptides in experimental diabetes; effects of treatment with nerve growth factor or brainderived neurotrophic factor. Mol Brain Res 21:171-175

33. Fernyhough P, Diemel LT, Brewster WJ, Tomlinson DR (1994) Deficits in sciatic nerve neuropeptide content coincide with a reduction in target tissue nerve growth factor messenger RNA in streptozotocin-diabetic rats: effects of insulin treatment. Neuroscience 62:337-344

34. Apfel SC, Arezzo JC, Moran M, Kessler JA (1993) Effects of administration of CNTF on normal motor and sensory peripheral nerves in vivo. Brain Res 604:1-6

35. Oates PJ (2002) Polyol pathway and diabetic peripheral neuropathy. Int Rev Neurobiol 50:325-392 
36. Calcutt NA, Muir D, Powell HC, Mizisin AP (1992) Reduced ciliary neuronotrophic factor-like activity in nerves from diabetic or galactose-fed rats. Brain Res 575:320-324

37. Thoenen H (1991) The changing scene of neurotrophic factors. Trends Neurosci 14:165-170

38. Curtis R, Adryan KM, Zhu Y, Harkness PJ, Lindsay RM, DiStefano PS (1993) Retrograde axonal transport of ciliary neurotrophic factor is increased by peripheral nerve injury. Nature 365:253-255

39. MacLennan AJ, Vinson EN, Marks L, McLaurin DL, Pfeifer M, Lee N (1996) Immunohistochemical localization of ciliary neurotrophic factor receptor alpha expression in the rat nervous system. J Neurosci 16:621-630

40. Kolta MG, Ngong JM, Rutledge LP, Pierzchala K, Van Loon GR (1996) Endogenous opioid peptide mediation of hypoalgesic response in long-term diabetic rats. Neuropeptides 30:335-344

41. Calcutt NA, Malmberg AB, Yamamoto T, Yaksh TL (1994) Tolrestat treatment prevents modification of the formalin test model of prolonged pain in hyperglycemic rats. Pain 58:413-420

42. Calcutt NA, Li L, Yaksh TL, Malmberg AB (1995) Different effects of two aldose reductase inhibitors on nociception and PGE. Eur J Pharmacol 285:189-197

43. Ahlgren SC, White DM, Levine JD (1992) Increased responsiveness of sensory neurons in the saphenous nerve of the streptozotocin-diabetic rat. J Neurophysiol 68:2077-2085
44. Khan GM, Chen SR, Pan HL (2002) Role of primary afferent nerves in allodynia caused by diabetic neuropathy in rats. Neuroscience 114:291-299

45. Suzuki Y, Sato J, Kawanishi M, Mizumura K (2002) Lowered response threshold and increased responsiveness to mechanical stimulation of cutaneous nociceptive fibers in streptozotocin-diabetic rat skin in vitro--correlates of mechanical allodynia and hyperalgesia observed in the early stage of diabetes. Neurosci Res 43:171-178

46. Pertovaara A, Wei H, Kalmari J, Ruotsalainen M (2001) Pain behavior and response properties of spinal dorsal horn neurons following experimental diabetic neuropathy in the rat: modulation by nitecapone, a COMT inhibitor with antioxidant properties. Exp Neurol 167:425-434

47. Chen SR, Pan HL (2002) Hypersensitivity of spinothalamic tract neurons associated with diabetic neuropathic pain in rats. J Neurophysiol 87:2726-2733

48. Freshwater JD, Svensson CI, Malmberg AB, Calcutt NA (2002) Elevated spinal cyclooxygenase and prostaglandin release during hyperalgesia in diabetic rats. Diabetes 51:2249-2255

49. Calcutt NA (2002) Potential mechanisms of neuropathic pain in diabetes. Int Rev Neurobiol 50:205-228

50. Dobretsov M, Hastings SL, Romanovsky D, Stimers JR, Zhang JM (2003) Mechanical hyperalgesia in rat models of systemic and local hyperglycemia. Brain Res 960:174183 